\title{
ADVANCES IN ANHLIYSIS AND CONTROL OF TIME-DELAYED DYNAMICAL SYSTEMS
}


This page intentionally left blank 


\title{
ADVANCES IN ANALYSIS AND CONTROL OF TIME-DELAYED DYNAMICAL SYSTEMS
}

\author{
Edited by \\ Jian-Qiao Sun \\ University of California-Merced, USA
}

\section{Qian Ding}

Tianjin University, China

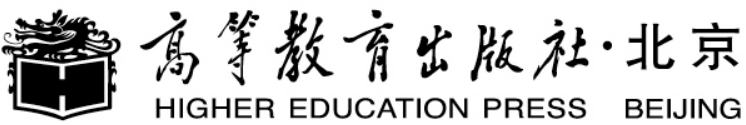


Editors

Jian-Qiao Sun

School of Engineering,

University of California-Merced

CA, USA
Qian Ding

Department of Mechanics

Tianjin University

Tianjin, China

Copyright (C) 2013 by

Higher Education Press Limited Company

4 Dewai Dajie, Beijing 100120, P. R. China and

World Scientific Publishing Co Pte Ltd

5 Toh Tuck Link, Singapore 596224

All rights reserved. This book, or parts thereof, may not be reproduced in any form or by any means, electronic or mechanical, including photocopying, recording or any information storage and retrieval system now known or to be invented, without written permission from the Publisher.

ISBN 978-981-4522-02-1

Printed in P. R. China 


\section{Preface}

Analysis and control of time-delayed systems have a large number of application areas including mechanical, control, transportation, economic, and biological systems. There has been a steady increase in the interest in time-delayed dynamic systems as demonstrated by a number of published articles on the subject shown in the figure below. This book presents a collection of papers presented at the International Workshop on Recent Advances in Analysis and Control of Time-delayed Dynamical Systems in Tianjin, China on July 2 to 4, 2012. The book takes a snap shot of this active research area, and contains the research work and survey articles from the world leading experts on analysis and control of dynamic systems with time delay.

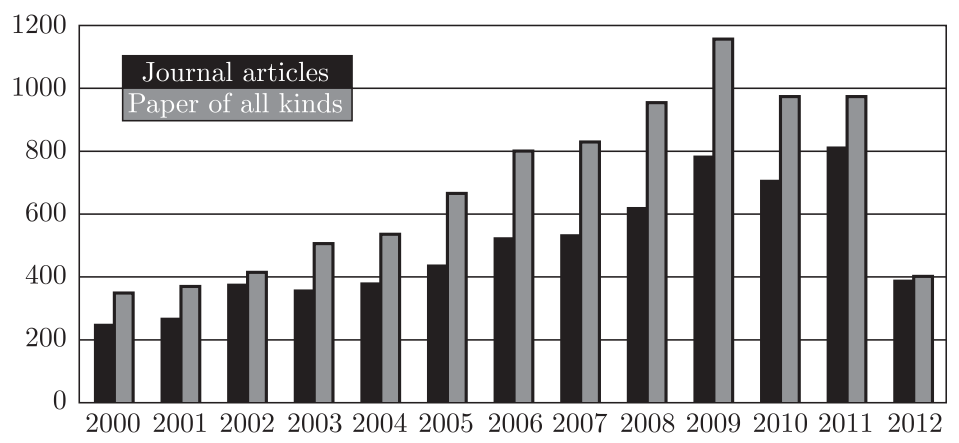

The trend of number of publications on dynamic systems with time delay in the past twelve years, based on a SCI search in June, 2012.

The chapters in the book cover a broad range of important topics. The complete quadratic Lyapunov stability conditions are discussed in Chapter 1, which offer necessary and sufficient conditions for stability of time-delayed dynamical systems. Chapter 2 describes recent advances in analysis and numerical methods of delayed differential equations (DDEs) with state-dependent time delay. State-dependent time delay can find important applications in complex dynamical systems, particularly in the systems with human interactions such as car following. Engineering applications of time-delayed dynamical systems are indeed plenty. Chapter 3 is focused on time-periodic time-delayed systems, and reports stimulating applications in machining process. Chapter 8 , on the other hand, presents a methodology study of the Chebyshev spectral continuous time approximation for estimation and 
control of time-delayed dynamical systems. We have organized a few chapters on complex dynamics of time-delayed systems including synchronization in complex networks (Chapter 4), delay induced resonances (Chapter 6), Hopf bifurcation of time-delayed systems with complex coefficients (Chapter 7), and noise-induced dynamics of time-delayed systems (Chapter 9). Control problems of time-delayed systems have received a great deal of attention in the literature. This is reflected in the chapters of the book. Chapter 5 presents stochastic optimal control of quasi integrable Hamiltonian systems with delayed feedback control. Chapter 10 presents an overview of theoretical and experimental research on analysis, control, and timedelayed identification of structural systems. Chapter 11 deals with switching controls of uncertain linear and nonlinear dynamical systems with time delay, including supervisory control and sliding mode control.

The topics covered by the chapters are of broad interests to the research community of dynamics and control. The book can therefore be a good reference to the researchers in the community of dynamics and control including mechanical, civil, structural, aerospace, naval, and electrical engineers, and can serve as a study guide to graduate students pursuing research in the area of dynamics and control with a special interest in time-delayed dynamical systems.

Jian-Qiao Sun

Merced, California

Qian Ding

Tianjin, China

November, 2012 


\section{Contents}

Chapter 1 Complete Quadratic Lyapunov-Krasovskii Functional: Limitations, Computational Efficiency, and Convergence

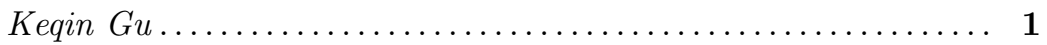

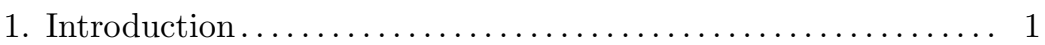

2. Complete Quadratic Lyapunov-Krasovskii Functional ........ 5

3. Discretized Lyapunov Functional Method $\ldots \ldots \ldots \ldots \ldots \ldots \ldots$

4. Coupled Differential-difference Equations ............... 11

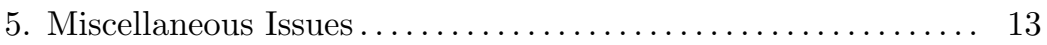

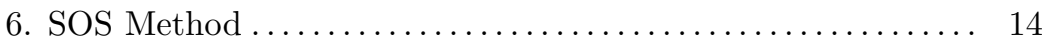

7. Conclusions and Perspectives ..................... 15

References .................................... 15

Chapter 2 Recent Approaches for the Numerical Solution of Statedependent Delay Differential Equations with Discontinuities

Alfredo Bellen................................... 21

1. Introduction.................................... 21

2. Weak Solutions .............................. 28

3. Regularization Techniques....................... 30

4. Comparing Regularizations ....................... 39

References ...................................... 39

Chapter 3 Engineering Applications of Time-periodic Time-delayed Systems

Gábor Stépán................................... 41

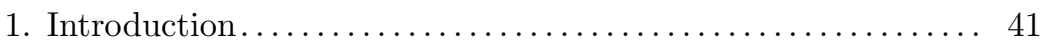

2. Delayed Mathieu Equation ....................... 42

3. Semi-discretization Method for Periodic DDEs............ 44

4. Engineering Applications .......................... 47

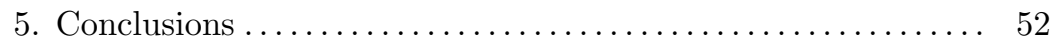

References .................................... 54

Chapter 4 Synchronization in Delay-coupled Complex Networks Eckehard Schöll................................ 57 


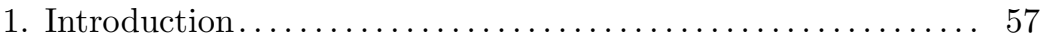

2. Stability of Synchronization for Large Delay ............ 58

3. Cluster Synchronization ........................... 63

4. Adaptive Synchronization ........................ 65

5. Transitions between Synchronization and Desychronization .. 71

6. Conclusion and Outlook ........................... 78

References ..................................... 79

Chapter 5 Stochastic Dynamics and Optimal Control of Quasi Integrable Hamiltonian Systems with Time-delayed Feedback Control

Weiqiu Zhu, Zhonghua Liu........................... 85

1. Introduction.................................. 85

2. Stochastic Averaging Method for Quasi Integrable Hamiltonian Systems with Time-delayed Feedback Control............ 86

3. Stochastic Dynamics of Quasi Integrable Hamiltonian Systems with Time-delayed Feedback Control .................... 98

4. Stochastic Optimal Control of Quasi Integrable Hamiltonian Systems with Time-delayed Feedback Control............ 142

5. Concluding Remark ............................ 159

References ....................................... 160

Chapter 6 Delay Induced Strong and Weak Resonances in Delayed Differential Systems

Jian Xu, Wanyong Wang........................... 163

1. Introduction .................................. 163

2. Analysis for Double Hopf Bifurcation............... 166

3. Conditions for Strong Resonances and Weak Resonances.... 173

4. Weak and Strong Resonances in Delayed Feedback Systems. 179

5. Weak and Strong Resonances in Van der Pol Systems with Delay Coupling.................................. 184

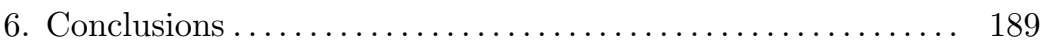

References ....................................... 189

Chapter 7 Stability and Hopf Bifurcation of Time-delayed Systems with Complex Coefficients

Zaihua Wang, Junyu Li ............................. 193

1. Introduction .................................. 193

2. The Crossing Direction for Stability Analysis ........... 195

3. Numerical and Graphical Stability Test............... 205

4. Pseudo-oscillator Analysis for Hopf Bifurcation........... 209

5. Conclusions .................................... 216 


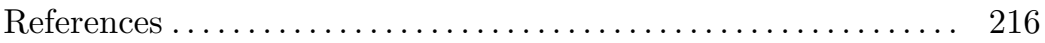

Chapter 8 Estimation and Control in Time-delayed Dynamical Systems Using the Chebyshev Spectral Continuous Time Approximation and Reduced Liapunov-Floquet Transformation

Eric A. Butcher, Oleg Bobrenkov, Morad Nazari,

Shahab Torkamani........................ 219

1. Introduction .............................. 219

2. Chebyshev Spectral Continuous Time Approximation... . . . 222

3. Reduced Liapunov-Floquet Transformation ............. 230

4. Feedback Control of Periodic Delayed Systems............ . 235

5. Stochastic State, Parameter, and Delay Estimation ........ 242

6. Application to Observer-based Delayed Feedback Control of Spacecraft Attitude ....................... 250

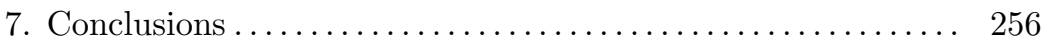

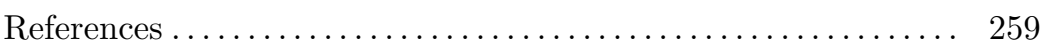

Chapter 9 Noise-induced Dynamics of Time-delayed Stochastic Systems

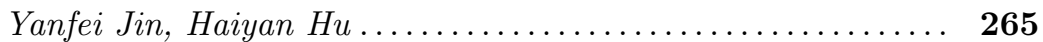

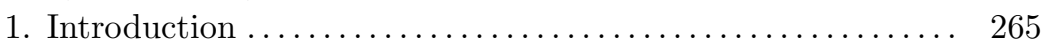

2. Fundamentals for Time-delayed Stochastic Systems ...... . . 266

3. Dynamical Behaviors of the Stochastic Systems with Time-delayed Feedback Control ................. 272

4. Noise-induced Resonances in Delayed Bistable Systems . . . . 294

5. Concluding Remarks ........................ 300

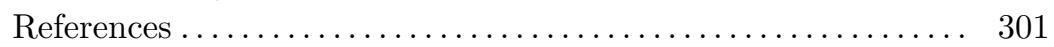

Chapter 10 Some Studies on Delayed System Dynamics and Control Guo-Ping Cai, Long-Xiang Chen, Kun Liu............. 309

1. Introduction .......................... 309

2. Time Delay Identification .................... 310

3. Two Time-delayed Controllers for Linear Structural Systems ............................. 311

4. Time-delayed Controller for Nonlinear Structural Systems . 313

5. Parameter Robustness of Time-delayed Controller ...... . . 315

6. Robust $\mathbf{H}_{\infty}$ Time-delayed Controller Based on The LMI

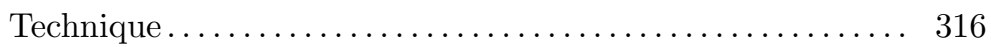

7. Delayed Positive Feedback Control Technique .......... 318

8. Time Delay Experiments ........................ 319

9. Concluding Remarks ........................ 321 
References .

\section{Chapter 11 Switching Control of Uncertain Dynamical Systems} with Time Delay

Jian-Qiao Sun, Xiao-Yan Zhang, Zhi-Chang Qin,

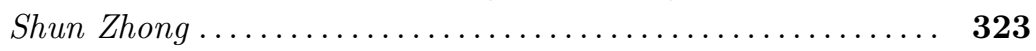

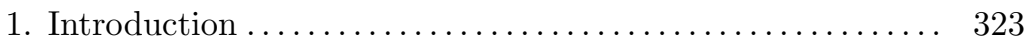

2. Supervisory Control for Systems with Uncertain Time

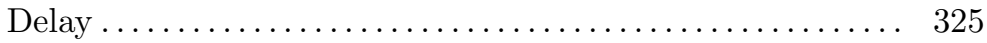

3. Sliding Mode Control Design for Uncertain Systems . . . . . 334

4. Concluding Remarks ........................... 339

References.................................... 340 